\title{
Performance Analysis and Optimization of Linear Restoration in Spatial Domain
}

\author{
Anil L. Wanare \\ RTM Nagpur University \\ B.D.C.O.E.Sevagram, India
}

\author{
Pratik D. Shah \\ G.H.R.I.E. \& T., Pune \\ University of Pune, India
}

\author{
Dilip D. Shah, PhD. \\ G.H.R.C.E.M., Pune \\ Principal \& ACM, \\ University of Pune, India
}

\begin{abstract}
This paper is concerned with critical performance analysis of spatial linear restoration techniques for still images from various fields (Medical, Natural and Arial images). The performances of the linear restoration techniques are provided with possible combination of various additive noises and images from diversified fields. Efficiency of linear restoration techniques according to difference distortion and correlation distortion metrics is computed. Tests performed on monochrome images, with various synthetic and real-life degradations, without and with noise, in single frame scenarios, showed good results, both in subjective terms and in terms of the increase of signal to noise ratio (ISNR) measure. The comparison of the present approach with previous individual methods in terms of mean square error, peak signal-to-noise ratio, and normalized absolute error is also provided. In comparisons with other state of art methods, our approach yields better to optimization, and shows to be applicable to a much wider range of noises. We discuss how experimental results are useful to guide to select the effective combination.
\end{abstract}

Keywords: Linear image restoration, Correlation distortion metrics, Wiener filter, Monochrome image denoising, Additive noise.

\section{INTRODUCTION}

The need for efficient image restoration techniques has grown with the massive production of digital images from various fields often taken in noisy conditions. So it is still exigent problem for researchers. The two main limitation in image accuracy are categorized as blur and noise, blur is intrinsic to image acquisition system [1] and second main image perturbation is the different type of noises. Linear restoration techniques deal with those images that have been recorded in the presence of one or more sources of degradation. Spatial domain is based conditionally on the values of the picture element in neighborhood under consideration and employs a low pass filtering on groups of picture elements the higher region of frequency spectrum [2]. The performance of an image linear restoration method depends on its ability to detect the presence of noisy picture element in the digital image. An interesting method for restoring of single type of image was proposed in [2] [3]. This method appears not to pose any strong restrictions on the degradation. In the cited paper, several experimental results on synthetic noises are shown, but little information is provided about them. From the information that is given, it appears that the degradation techniques that were used in the experiments either circularly symmetric or corresponded to straight line motion blurs. There seems to be no reason for the method not to be able to successfully deal with other kinds of noises, however the PSF, Gaussian, Speckle, Salt \& pepper, and Poisson noises are shown in this paper appear to have different density. The improvements in increase signal to noise ratio (ISNR) seem to be between particular ranges in $\mathrm{dB}$ for specific standard deviation. The experimental result presented in section IV show that, with much at particular density, our scheme at normally yielded to optimum selection according to larger improvement in linear restoration methods. In all cases, one has access to more than one degraded image from the same noise a fact which can be used to the ill-posedness of the problem. The scheme also assumed that the quality of the original image, before the degraded, as happens in most images from various fields. To the author's acknowledge, this is first scheme to be proposed, which is able to yield result of optimum selection in such a wide range of situations. The performance and robustness of the linear restoration techniques were tested in various experiments, with synthetic and real life degradation without and with density of noises on the restoring filters, using monochrome images, and under the single frame. The quality of the results was evaluated both visually and in terms of ISNR, normalized mean square error (NMSE). Detailed comparisons of wiener and mean filtering (linear restoration) with MSE, PSNR difference distortion metrics were evaluated, and show that the proposed scheme yields significantly better for optimum selection of restoration technique

The remainder of the paper is organized as follows: Section II provides a background review on the concepts involved in linear restoration and modeling different types of noise in brief. The wiener filter and mean filter implementation in section III. In section IV, results obtain from the same. In section V \& VI; concluding remarks, discussion and future research directions.

\section{BACKGROUND REVIEW}

Contaminated image model considered for analysis as equation, $g=h f+n$, where $\mathrm{g}, \mathrm{f}$ and $\mathrm{n}$ are $\mathrm{MN}$ dimensional vectors and ' $h$ ' is (MNxMN) block circulant matrix represents degradation process embedded in continuous tone image formation process, ' $\mathrm{g}$ ' vector from the given matrix $\{g(r, c)\}$ is $g(r, c) \in\{0,1,2,3,4, \ldots . . L .-\quad$.We have considered spatial degradation models for analysis are commonly i)Atmospheric turbulence ii)Motion blur iii)Defocused system. Space-invariant point spread function for mentioned types are described in details Space- invariant point spread function for mentioned [6] [11]. All noises occur in spatial domain modeled as Gaussian, Speckle, Poisson, and Salt \& Pepper. According to application Gaussian noise is distributed over signal while transmission which has bell shaped PDF [8]. Salt \& Pepper is an impulse noise, it is generally caused by malfunctioning in picture element in common CCD and CMOS sensors, faulty memory 
location or timing problem in quantization process, transmission line is not installed properly[8][9]. Speckle noise occurs in almost all coherent imaging system such as laser acoustic and synthetic aperture radar imagery. The Poisson distribution is a one parameter of discrete distribution that takes nonnegative integer value. The parameter, both the mean and variance of the distribution is explained in [10].

\section{IMAGE RESTORATION}

\subsection{Adaptive Wiener and Mean Filtering}

Wiener filter is a class of optimum linear estimation of a desired image. The statistical approach is to the solution of linear restoration, statistical parameters of the use signal and unwanted additive noise. A useful approach of optimization problem is to minimize the mea-square value of the error. The introduction of noise into an image is often modeled as an additive process [11]. Mask is rotated on each pixel of image in order to obtain the response; it is being obtained by the sum of product of the mask coefficients with the corresponding pixels directly under the mask. $\hat{P}(x, y)=\sum w(x, y)[q(x, y)-m(x, y)]^{2}$ Minimum , where $w(x, y)$ is response of linear restoration technique, $m$ and $q$ are noisy image, output respectively. The mean filtering is to replace each pixel value in an image with mean value of its neighbors, including itself [12]. This has the effect of eliminating pixel value which is unrepresentative of their surroundings. Mean filter is totally based on convolution operation of linear algebra. The same operation is based around a kernel which is known as mask. Often a $3 \times 3,5 \times 5$, $7 \times 7$ square kernels can be used for smoothing according to the requirement. Mean filtering categorized into Harmonic, Geometric and Contra-harmonic techniques. Restored pixels can be calculated by equation 01 [13].

$$
\hat{p}(x, y)=\frac{m n}{\sum_{(s, t) \in S_{x, y}} \frac{1}{g(s, t)} \ldots \ldots \ldots \ldots \ldots . . .01}
$$

In contra-harmonic mean restored pixel with help of order of the linear restoration filtering technique. And it can be expressed as equation 02 .

$$
\hat{p}(x, y)=\frac{\sum_{(s, t) \in S_{x, y}} g(s, t)^{Q+1}}{\sum_{(s, t) \in S_{x, y}} g(s, t)^{Q} \ldots \ldots \ldots \ldots \ldots . . .02}
$$

Where $g(x, y)$ noisy image and $(s, t)$ coordinates of degraded pixels [13].

\subsection{Quality measures and Problem formulation}

Image dependent noise can be estimated form multiple frames or single image. Estimation from single frame is an under constrained problem and further assumption have to be made for degradation. In the image restoration literature degradation often assumed to be an AWGN. A widely used estimation techniques are based on the mean absolute deviation [14].In [15] quantitative measures are estimated for each intensity in spatial domain. A Stefano and P. Whites, system was based training samples in other domain only for natural images [16].Generalization expectation maximization restoration techniques in any domain developed and estimate the spectral features. The scheme for restoration is followed by synthetic degradation to give the optimum solution of noisy images from diversified fields. Signal dependent is estimated from the smooth region of the image by segmenting the image gradient with an additive threshold in the different domain for specific type of image [17]. By comparing the scheme which is proposed optimization up to some extend for synthetic degradation and real life degradation to diversified field images. And scheme provides a principled way for selecting linear restoration methods for estimating the quality of noisy images under various densities of degradations.

Subjective measure that we used for evaluating the quality of result of restoration tests is mean square error (MSE). However, the computation meaningful increase signal to noise ratio (ISNR) in restoring situation raises some spatial issues that we now address. We start by recalling basic concept of ISNR, the noise of ' $y$ ' as $\left(y-x_{0}\right)$, and the noise of ' $x$ ' as $\left(x-x_{0}\right)$. ISNR of restored image ' $x$ ' relative to the degraded image ' $y$ ' is, then, the difference between the SNR of ' $x$ ' and SNR of ' $y$ '. It can be computed, in decibels, as

$$
I S N R=10 \log _{10} \frac{\sum_{i}\left(y^{i}-x_{0}{ }^{i}\right)^{2}}{\sum_{i}\left(x^{i}-x_{0}{ }^{i}\right)^{2}} \ldots \ldots . .3
$$

Where the superscript ' $i$ ' indexes the image pixel, and sums run through all pixels. The special issues arises in the computation of this measure in restoration situation, are due to the following. The restoration problem is strongly ill-posed. This means that non-regularized solution have a large degradability. There are four kind of degradation that occurs are mentioned in section 2. These degradations should be taken into account by the quality measures. MSE between the restored image and the original, then we would take the difference between two images element-by-element, square the result and PSNR can be estimated by

$$
P S N R=10 \log _{10}\left(\frac{\left(2^{n}-1\right)^{2}}{M S E}\right) \ldots \ldots . .4
$$

\section{EXPERIMENTAL RESULTS}

The main experiment has been performed with on both synthetic and real life degraded gray scale images to test linear restoration methods. Each image was degraded with Gaussian, Poisson, Speckle and Salt \& Pepper with PSF.

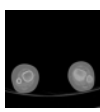

a.apperts

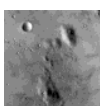

f.plannet

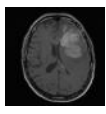

b.brain

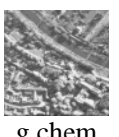

g.chem

plant

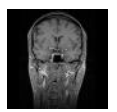

c.bone

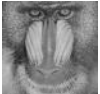

d.baboon

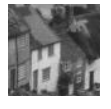

e.house
Fig1: set of typical gray scale images used in experiment.

All images from Medical, Natural and Arial images are applied for experimentation shown in fig.1. We tested the linear restoration techniques on the same images. Wiener and Mean filters implemented in MATLAB Version 7.12.0.635. 
Especially goals are to examine the correlation quality between numerical results with ground truth data image (corrupted by additive noise) and results from experiments with original image and to quantify the performance in percentage according to level of noise. Details parameters were computed on Intel core 2 duo systems at $2.8 \mathrm{GHz}$, for monochrome 33 images of size $256 \times 256$ as some of them shown in fig.1. Experiments are performed to observe the effectiveness of the linear restoration techniques. The qualities of the various field images are compared in terms of visual quality and correlation parameters.

Table 1. Comparison of the result obtained after restored image on wiener filter, each entry gives the improvement in (\%) according to MSE

\begin{tabular}{|c|c|c|c|c|}
\hline Image & Gaussian & Speckle & Poisson & $\begin{array}{c}\text { Salt \& } \\
\text { Pepper }\end{array}$ \\
\hline Apperts & 11.29 & $\mathbf{6 0 . 7 8}$ & 13.30 & 47.70 \\
\hline Brain & 12.41 & 44.81 & 7.24 & $\mathbf{7 9 . 7 7}$ \\
\hline Bone & 12.23 & 46.19 & 12.35 & $\mathbf{7 7 . 7 1}$ \\
\hline Baboon & 3.57 & $\mathbf{5 7 . 1 6}$ & 33.59 & 27.34 \\
\hline House & 4.06 & 33.66 & $\mathbf{6 2 . 7 9}$ & 17.50 \\
\hline Plannet & 11.64 & $\mathbf{6 8 . 9 9}$ & 21.09 & 02.35 \\
\hline $\begin{array}{c}\text { Chem. } \\
\text { Plant }\end{array}$ & 12.07 & $\mathbf{5 6 . 8 0}$ & 07.67 & 36.56 \\
\hline
\end{tabular}

Table 1 gives a summary of the result, in terms of improvement in percentage according to Mean Square Error to different types of synthetic degradation. Improvement is calculated by the ratio of difference between mean square error of original image \& noisy image and restored images to the MSE between original \& noisy image.

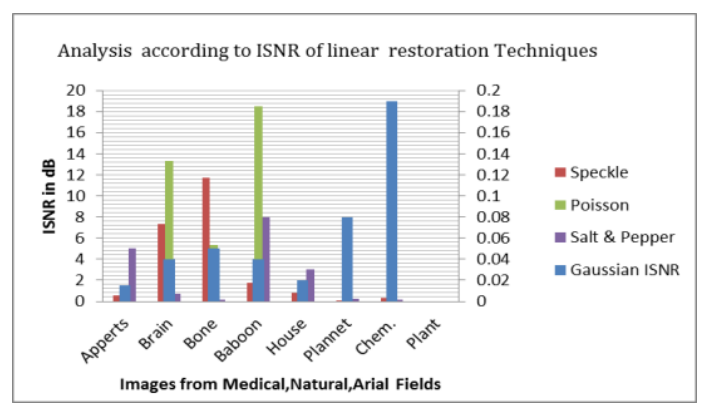

Fig 2: ISNR values for linear restoration method with synthetic degradations to sequence of images

In fig 2 shows comparison of ISNR values obtained and it gives clearly significant improvement in quality result. Linear restoration technique only yielded a significant improvement in image quality to the natural and medical images for Poisson noise. In fact, these ISNR values are relatively closed to the values attained by state of the art restoration techniques. Figure 3 shows adaptive wiener filter providing significant result according to the values of PSNR for Poisson, Speckle and Salt \& Pepper to X-Ray images. Average mean filter gives $33.50 \mathrm{~dB}$ for Gaussian. Geometric mean filter is providing the significant result for Poisson and Speckle noises. AWF \& AM are providing PNSR more than $30 \mathrm{~dB}$ for MRI and CT images. For natural images Arithmetic Mean for Gaussian and Adaptive Wiener Filter for Speckle, Poisson, Salt \& Pepper noises. In Arial filed AWF and AM, Geometric Mean are providing significant PSNR to Gaussian, Speckle and Salt \& Pepper noises respectively.
Besides testing the proposed scheme on synthetic degradations, also applied it to real life degradation. We used some gray sale images degraded by camera itself due to faulty CCD and CMOS sensors. The results obtained with these images were lower visual quality than those obtained with synthetic degradations. The noise produced by CCD, CMOS and climate condition is not synthetic degradation and its intensity independent from the image intensity, so that restoration method not showing the response as to synthetic degradation [17]

\section{DISCUSSION}

Stress on that, although our numerical result of linear restoration methods with different types of noises, some quantitative measure are crucial: MSE, PSNR, and ISNR. In fact experiments have shown that the same sequence of images and numerical parameters yield considerable results for wide range of images from Medical, Natural and Arial fields. This being said, we should note that, by considering quantitative measures, somewhat better optimum selection can obtain without wasting time to perform experiment separately. In several practical applications, it may quite possible to select restoration methods to particular field images. The choice of restoration techniques for diversified field images degraded by different noises with density is crucial for selection. The selection of the same for specific noise and field has been provided for researchers, it is some optimum solution to select the linear restoration techniques.

\section{CONCLUSION}

We have presented a critical performance of linear restoration techniques for noisy images from diversified fields. The techniques are handled by synthetic degradations to compute the numerical results. We have computed the parameters to the evaluation of linear restoration performance of linear restoration techniques. According to the restoration quality of linear methods were visually and quantitatively compared and provided optimum solution for the selection to particular field image.

Experimental performance showed on a variety of images, only on gray scale, with and without synthetic noises in single image at a still situation. We compared performance of linear restoration methods, adaptive wiener filter providing significant result according to the values of PSNR for Poisson, Speckle and Salt \& Pepper to Medical images. Average mean filter gives significant PSNR for Gaussian. Geometric mean filter is providing the significant result for Poisson and Speckle noises. Adaptive Wiener Filter \& Arithmetic Mean are providing PNSR more than thirty in $\mathrm{dB}$ for MRI and CT images. For natural images Arithmetic Mean for Gaussian and Adaptive Wiener Filter for Speckle, Poisson, Salt \& Pepper noises. In Arial filed AWF and AM, Geometric Mean are providing significant PSNR to Gaussian, Speckle and Salt \& Pepper noises respectively. So far, whenever the degradation image has noise, the particular restoration has to be choosing, by selecting the linear restoration method which yields the best compromise between types of image from particular field

and noise detail. After performing with thirty three images from different fields, we can't recommend strongly to use particular restoration methods is suitable for particular type image with specific degradation. An automatic selection criterion will obviously be useful. This is the direction in which further research will be done. 


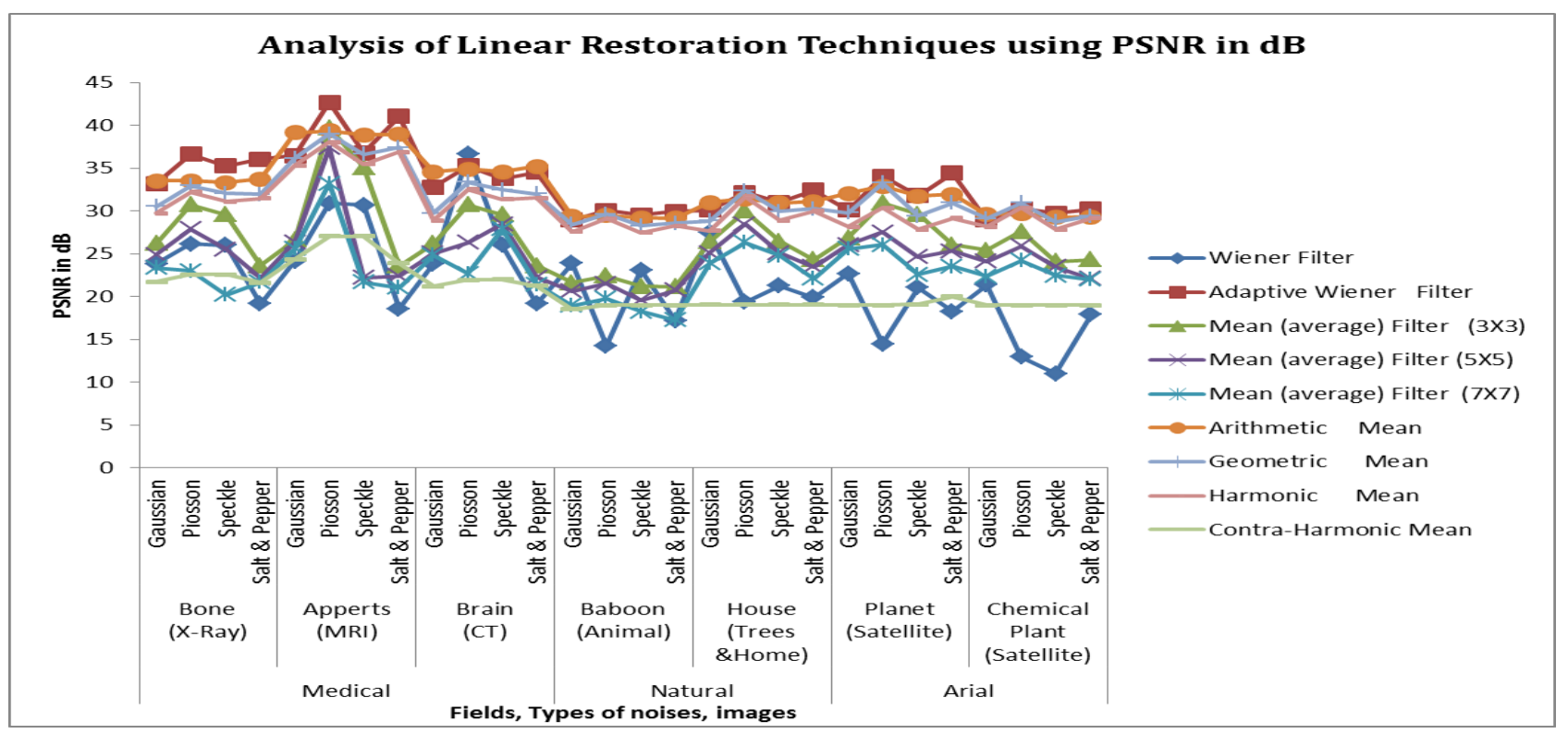

Fig 3: PSNR Values in dB obtained from Linear Restoration Techniques with Different Noises to Diversified Field Images

\section{ACKNOWLEDGEMENT}

The authors wish to thank the anonymous reviewers. The author would like to thanks of research center of BDCOE, RTM Nagpur University, Nagpur, INDIA. The authors gratefully acknowledge the support from research center of University of Pune, for research grant to work on this research scheme.

\section{REFERENCES}

[1] T.Weissman, E,Ordentlich, G.Seorussi, S.Verdi, and M.J.Weinberger, "Universal discrete denoising :known channel,” IEEE trans. Info. Theory, Jan.2005.

[2] A. Buades, B.Coll, and J.M.Morel, " A new image denoising, with new one," SIAM of multiscale Modeling (MMS)

[3] Anil L. Wanare, Dr. Dilip D. Shah, " Performance Analysis and Optimization of Nonlinear Restoration Techniques in Spatial Domain," International Journal of Image Processing, Volume 06, Issue 02,pp 123137,April. 2012

[4] T.Weissman,E. Ordentlich, S. Verdi, and M.J.Weinberger, "A discrete universal denoiser and its application," in proc. Of ICIP'2003, Barcelona, Sept.2003.

[5] A.kubota, K.Aiaiwa, "A reconstruction arbitrarily focused images from two diff. focused images using linear filters," IEEE trans.IP, Vol. 14,no. 11, Nov.2011..

[6] S.chang, S.W.Yun, and P. Prak, "PSF search algorithm for dual exposer type blurred," IEEE Trans., SP, Vol.15, no.02, Feb.2003.

[7] M.Blume, D.Zikic, W.Wain, and N. Nawab, "A new and general methods for blind SV deconvolution of biomedical images," in proc. MICCAI, 2007, pp.743750 .

[8] D. Kundur, D. Hatzinakos, "Blind image deconvolution," IEEE Signal Processing Mag., pp46-64, May1996.
[9] M. Sindhant Devi, V. Radhika "Comparative approach for speckle reduction in medical images," International Journal of ART, Vol.01, Issue 01, pp-7-11, 2011.

[10] J. Umamaheshwari, "Hybrid denoising methods for mixed noise in medical images," IJACSA, Vol.03, no. 05 pp-44-47, 2011

[11] Mariana S.C Almeida and Luis B. Almeida, "Blind and semi blind deblurring of natural images," IEEE Trans. Vol.19, no.01, Jan. 2011

[12] R.Deriche, "Fast algo. For low level vision," IEEE trans. MI, vol.12,no. 01, pp. 78-87, Jan. 1990.

[13] Anil K. Jain, "fundamental of digital image processing." Englewood Cliffs, New Jersey, 1989.

[14] Peter J. Rausseeuw, and Christiph Croux, " altenative to the median absolute deviation" Journal of American statistical association," vol. 88, no. 424, pp 1273-1283.

[15] Tang Sang cho, Sing bing Kang, "Image restoration by matching gradient distribution," IEEE trans.

[16] Jean Tarel, Nicolas, "Fast visibility restoration from a single gray level images," LCPC-INRETS (LEPSIS) , Paris France.

[17] Srinivasa and K. Nayar, "Contrast restoration of weather degraded images," IEEE Trans. PAMI, Vol. 25, No. 06.

[18] M. D Grassberg and S.K.Nayar, "Modeling the space response function of camera," IEEE trans. PAMI,vol. 26. Oct. 2004.

[19] D. Donoho, "Denoising by soft thresholding," IEEE trans IT, vol. 03, 1995.

[20] J Astola and P. Kaosmanen, fudamentals of nonlinear and linear filtering, Boca Raton, CRC press, 1997.

[21] L.Yin, R. Yang M Gabbouj, and Y Neuvo, "Weighted median filtering for speckle suppression in medical imaging," IEEE trans, CS, vol. 36. 
[22] A. Stefano, P.White, "training method for image noise level estimation on wavelet component," JASP, vol.16,2004.

[23] T.Chen, K.K.Ma, and L.H.Chen, "Tri state median filter for denoising," IEEE Trans. IP. Vol.8, pp.1834-1838.

[24] Jong Sen Lee, "refined filtering of image noise using local statistics," Computer Graphics and Image Processing, Vol.15, issue 04,pp.380-89.

[25] Chang Shing Lee, You Thang kuo, "Fuzzy set and system," Vol.89, issue 02, pp. 157-180.

[26] E.Areu, M. Lightstone, S.K.Mitra, and K.Arakawa, "A new efficient approach for the removal of impulse noise from highly corrupted images," IEEE Trans. IP, Vol.05, no06,pp 1012-1025.

[27] E.Abreu, "Signal dependent rank-ordered Mean filters," Linear-Nonlinear Image processing, S.K.Mitra, Academic Press, 2000.

[28] P.S.Heckbert, "Filtering by repeated integration," in proc. Int. conf. CGIT, 1986, vol20, no.04,pp. 315-321.

[29] Arrate Munoz-Barrutia, Michel Unser, "Fast SpaceVarient Elliptical Filtering using Box Splines," IEEE Trans, IP, Vol.19, No.9, Sept.2010.pp. 2290-2305. 